Bundesgesundheitsbl - Gesundheitsforsch Gesundheitsschutz 2008 $\cdot 51: 583-584$

DOI 10.1007/s00103-008-0595-5

C) Springer Medizin Verlag 2008

\author{
B.-M. Kurth ${ }^{1} \cdot$ U. Koch ${ }^{2}$ \\ ${ }^{1}$ Robert Koch-Institut Berlin, BRD . ${ }^{2}$ Universitätsklinik Hamburg-Eppendorf, BRD
}

iebe Leserin, lieber Leser,

für insgesamt 38,7\% der 17.641 Kinder und Jugendlichen im Alter von o bis 17 Jahren, die am Kinder- und Jugendgesundheitssurvey (KiGGS) teilgenommen haben, wurde von den Eltern mindestens ein chronisches Gesundheitsproblem berichtet. Gleichzeitig wurde der aktuelle Gesundheitszustand für 93,1\% der Probanden als „sehr gut“ bzw. „gut" eingeschätzt. Nur etwa ein Viertel der Kinder mit einem chronischen Gesundheitsproblem hat nach subjektiver Einschätzung der Eltern einen erhöhten Versorgungsbedarf (Scheidt-Nave et al. in diesem Heft). Sind dies Widersprüche, Fehler in der Erfassung oder ein Spezifikum der Bevölkerungsgruppe der unter 18-Jährigen?

Dieser Frage wird in mehreren der in diesem Themenheft „Chronisch kranke Kinder"veröffentlichten Arbeiten nachgegangen. Der Einstiegsartikel von Schmidt und Thyen befasst sich mit möglichen Definitionen des chronisch Krankseins bei Kindern und Jugendlichen. Dabei wird deutlich, dass ein Kind nicht allein aufgrund des aktuellen Vorhandenseins eines chronischen Gesundheitsproblems als ,chronisch krank" klassifiziert werden kann. Weitere Informationen zu Dauer und Schweregrad der Erkrankung, zum daraus resultierenden Versorgungsbedarf sowie zu den Auswirkungen auf die Lebensqualität und die psychosozialen Bewältigungsstrategien las-
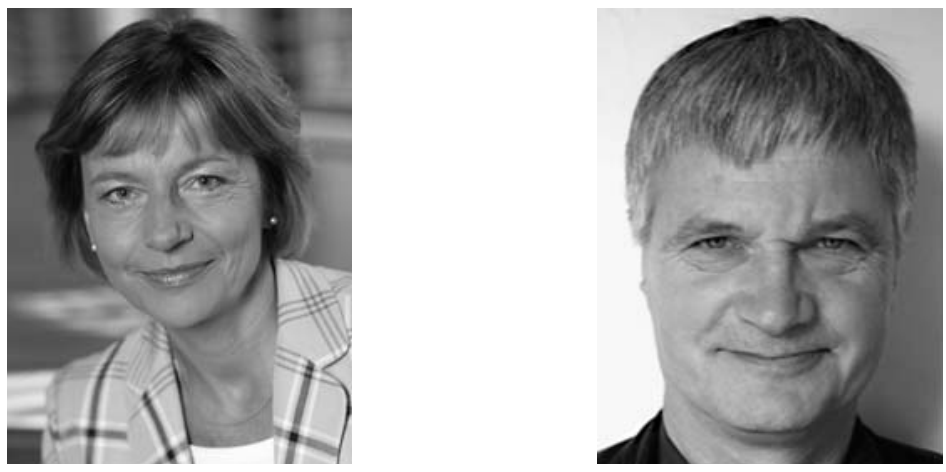

sen das chronisch Kranksein eines Kindes jeweils in einem anderen Licht erscheinen.

Daraus ergeben sich hohe Ansprüche an die Diagnostik einer chronischen Erkrankung im Kindes- und Jugendalter. Diese erfordert sowohl umfassende diagnostische Kenntnisse wie auch hinreichende klinische Erfahrungen. Exemplarisch zeigt dies die Arbeit von Huss, der sich mit der Aufmerksamkeitsdefizit-/Hyperaktivitätsstörung (ADHS), einer der häufigsten Verhaltensstörungen im Kindes- und Jugendalter, befasst. Im Rahmen einer umfassenden krankheitsspezifischen Diagnostik sind regelhaft die aktuellen und vergangenen Probleme, die Psychopathologie, die kognitive Leistungsfähigkeit, die somatisch-neurologischen Bedingungen und die sozialen wie emotionalen Konsequenzen wie auch Kompetenzen systematisch zu erfassen. Wichtig ist darüber hinaus die Erfassung der häufig auftretenden Komorbiditäten wie Legasthenie, Tic-Störungen oder Angsterkrankungen.

Der Umstand, dass je nach gewähltem diagnostischem Zugang und Verständnis in der internationalen Forschungslandschaft als auch im nationalen gesundheitspolitischen Kontext recht unterschiedliche Definitionen und Aussagen zum Anteil chro- nisch kranker Kinder in der Bevölkerung zu finden sind, erscheint zunächst nur theoretisch von Bedeutung zu sein. Mit der Public-Health-Zielstellung allerdings, möglichst vielen (am besten allen) Kindern unabhängig von vorhandenen Krankheiten optimale gesundheitliche Versorgung, fördernde psychosoziale Rahmenbedingungen und maximale Hilfestellungen zur Bewältigung von Belastungssituationen angedeihen zu lassen, wird die Frage nach dem chronisch kranken Kind sehr konkret und bekommen die Arbeiten der Autorinnen und Autoren dieses Heftes eine deutliche Anwendungsorientierung. So wird im Artikel von Hölling et al. zu Schutzfaktoren und Lebensqualität von Kindern, die unter Asthma, Adipositas oder ADHS leiden, unter Nutzung der KiGGS-Daten festgestellt, dass sich für an Asthma erkrankte Kinder auf Bevölkerungsebene kaum Beeinträchtigungen der gesundheitsbezogenen Lebensqualität feststellen lassen. Dies wird mit der Existenz sehr wirksamer Behandlungsmethoden und Bewältigungsstrategien erklärt. In der Arbeit von Petermann und Schauerte findet sich hierfür eine überzeugende Bestätigung: Durch Maßnahmen zur Förderung der Compliance von asthmakranken Kindern und deren Familien, be- 
schrieben als „optimiertes Asthmamanagement", kann die Lebensqualität der betroffenen Kinder nachweislich so verbessert werden, dass sie sich von der eines gesunden Kindes kaum noch unterscheidet.

In der Untersuchung von Petersen et al. beschreiben die Eltern von Kindern und Jugendlichen nach Schädel-Hirn-Trauma ein ähnlich positives Bild der Lebensqualität. Darüber hinaus berichten sie eine hohe Behandlungszufriedenheit. Ein in der Region vorhandenes kindgerechtes Versorgungsangebot erwies sich dabei als wichtiger Prädiktor für die Behandlungszufriedenheit der Eltern.

Die Untersuchung der Lebensqualität von jungen Patienten mit Hämophilie in der Arbeit von Bullinger et al. zeigt wesentliche Unterschiede in den Auswirkungen dieser Erkrankung im europäischen Vergleich. Diese Unterschiede geben Anlass zur Untersuchung der Ursachen hierfür und können in Empfehlungen zum Umgang mit dieser Krankheit münden.

Ein adipöses Kind, das unterschiedliche Versorgungsangebote des Gesundheitssystems in Anspruch nehmen kann, wird nicht immer auch mit Verbesserungen seines Gesundheitszustandes und der gesundheitlichen Lebensqualität rechnen können. Im Beitrag von Kliche und Mann werden verschiedene Versorgungsangebote für adipöse Kinder aufder Grundlage eines expertengestützten entwickelten Katalogs von Qualitätsmerkmalen bewertet. Hier wird deutlich, dass die für die Versorgung der betroffenen Kinder und Jugendlichen eingesetzten Res- sourcen in vielen Fällen keine guten Voraussetzungen bieten und deshalb als wenig Erfolg versprechend gelten müssen. Im Interesse der betroffenen Kinder sind hier Maßnahmen zur Qualitätsverbesserung zu treffen. Die Autoren unterbreiten Vorschläge hierfür.

Dass chronisch kranke Kinder in Abhängigkeit vom Schweregrad der Erkrankung und den bestehenden Behandlungsmöglichkeiten eine psychosoziale Belastung für die zugehörigen Familien darstellen, ist durch zahlreiche Publikationen belegt. Dass aber auch die umgekehrte Situation, dass Kinder, die mit einem chronisch erkrankten Elternteil aufwachsen, unsere Aufmerksamkeit verdienen, wird im Beitrag von Möller et al. sehr überzeugend dargelegt. Hier sind Hilfsangebote für Eltern und Kinder erforderlich, um chronische Gesundheitsprobleme der Kinder, die meist psychischer Natur sind, vermeiden zu helfen. Dieser präventive Ansatz stellt einen wichtigen Beitrag zur Erhaltung der psychischen Gesundheit betroffener Kinder dar.

Selbst wenn die WHO-Definition zur Gesundheit manchem unserer Leserinnen und Leser überstrapaziert erscheint, so ist der Zustand „vollkommenen physischen, psychischen und sozialen Wohlbefindens" aller Kinder, auch derjenigen mit einer chronischen Krankheit, erstrebenswert und überwiegend auch erreichbar, wie die Arbeiten in dieser Ausgabe des Bundesgesundheitsblattes partiell belegen. Die Vision von „chronisch gesunden Kindern“ in unserer
Gesellschaft kann damit als Zielorientierung aller medizinischen, präventiven, gesundheitspolitischen und individuellen Maßnahmen dienen, auch wenn sie wohl immer eine Vision bleiben wird.

Ihre
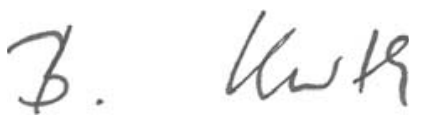

Bärbel-Maria Kurth

Ihr

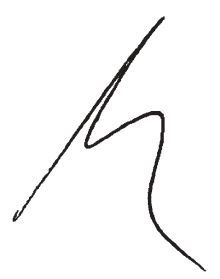

Uwe Koch

\section{Korrespondierende Autoren}

\section{Dr. Bärbel-Maria Kurth}

Robert Koch-Institut

Abteilung für Epidemiologie und

Gesundheitsberichterstattung

Postfach 650261

13302 Berlin, BRD

E-Mail: KurthB@rki.de

\section{Prof. Dr. Dr. Uwe Koch}

Universitätsklinikum Hamburg-Eppendorf Abt. für Medizinische Psychologie

Martinistraße 52

Pav. 69

20246 Hamburg, BRD

E-Mail: koch@uke.uni-hamburg.de 